\title{
Reconhecimento e uso de plantas medicinais pelos idosos do Município de Marmeleiro - Paraná.
}

\author{
BALBINOT, S. ${ }^{1}$; VELASQUEZ, P.G. ${ }^{1}$; DÜSMAN, E. ${ }^{*}$ \\ ${ }^{1}$ Universidade Paranaense - UNIPAR - Campus Francisco Beltrão - Paraná - Brasil. Av. Julio Assis Cavalheiro, \\ 2000 - Bairro Industrial - Cep: 85601-060 - Francisco Beltrão - PR. ${ }^{2}$ Universidade Estadual de Maringá - UEM \\ - Maringá - Paraná - Brasil. Avenida Colombo, 5790, Bloco H67, sala 11, Jardim Universitário, CEP: 87020-900 \\ - Maringá - Paraná - Brasil. *lisdusman@yahoo.com.br
}

RESUMO: O consumo de plantas medicinais tem base na tradição familiar e tornou-se prática generalizada na medicina popular. Neste sentido, este trabalho teve como objetivo verificar o grau de reconhecimento e uso de espécies medicinais utilizadas por 35 idosos do município de Marmeleiro - Paraná. Os resultados das entrevistas mostraram que todos os idosos conheciam, das vinte plantas medicinais apresentadas, a babosa, a camomila, a erva-cidreira, a macela, a malva, a manjerona, a noz-moscada, a pata-de-vaca e a sálvia. Além disso, 94\% dos mesmos faziam o uso de plantas medicinais, sendo que $71,4 \%$ destes, utilizavam frequentemente, enquanto $94,2 \%$ foram influenciados principalmente pelos familiares (pais e avós). As plantas medicinais consumidas diariamente pelos idosos eram a erva-cidreira (48,6\%), a malva $(34,3 \%)$, o quebra-pedra (34,1\%), a sálvia $(25,7 \%)$, a camomila $(22,9 \%)$, o guaco $(20 \%)$, a carqueja e a macela $(14,3 \%)$, a pata-de-vaca e o alecrim $(8,6 \%)$ e a babosa $(2,9 \%)$, preparadas na forma de chá (decocção) ou infusão. Vale destacar que a forma de obtenção das plantas medicinais era principalmente em plantações próprias $(88,6 \%)$. Desta forma, informações acerca das plantas medicinais devem retornar para a população, em especial a mais idosa, enfatizando o modo de cultivo e colheita, através de técnicas desconhecidas por eles, bem como a melhor forma de utilização de cada espécie medicinal, estimulando seu uso, com maior eficacia e segurança.

Palavras-chave: Etnofarmacologia, Medicina Tradicional, Uso de Plantas Medicinais por Idosos.

\begin{abstract}
Recognition and use of medicinal plants by the elderly in the city of Marmeleiro - Paraná. The use of medicinal plants is based on family tradition and became a common practice in popular medicine. Therefore, this study aimed to verify the level of recognition and use of medicinal plants used by 35 elderly in the city Marmeleiro - Paraná. The results of the interviews showed that all elderly knew, from twenty medicinal plants presented, aloe vera, chamomile, lemon balm, camomile, mallow, marjoram, nutmeg, paw-of-cow and sage. Besides that, $94 \%$ of them used medicinal plants, $71.4 \%$ of these frequently, influenced mainly by the family (parents and grandparents) $(94.2 \%)$. Medicinal plants consumed daily by the elderly were the lemon balm (48.6\%), mallow (34.3\%), shatterstone (34.1\%), sage $(25.7 \%)$, chamomile $(22.9$ $\%)$, guaco $(20 \%)$, gorse and camomile (14.3\%), paw-of-cow and rosemary $(8.6 \%)$ and aloe vera $(2.9 \%)$, prepared as tea (decoction) or infusion. We should note that the form to obtain the plants was mainly from their own gardens $(88.6 \%)$. We conclude that the information about medicinal plants should be available to the public, especially the elderly, emphasizing the methods of cultivation and harvesting, with techniques still unknown to them, and the best way to use each medicinal species, encouraging their use, but with no damage to health.
\end{abstract}

Keywords: Etnofarmacologia, Medicina Tradicional, Uso de Plantas Medicinais por Idosos.

\section{INTRODUÇÃO}

As plantas produzem substâncias químicas que podem atuar beneficamente ou agirem de forma tóxica sobre outros organismos. Portanto, para que o homem possa fazer uso medicinal de uma espécie vegetal com segurança, é necessário que a mesma seja estudada sob o ponto de vista químico, farmacológico e toxicológico (Ritter et al., 2002).

O consumo de plantas medicinais tem base na tradição familiar e tornou-se prática generalizada na medicina popular, sendo considerada uma terapia complementar ou alternativa para a promoção da saúde (Loya et al., 2009). O conhecimento das 
recomendações terapêuticas das plantas medicinais é, normalmente, uma característica presente na população mais idosa, sendo que estas plantas são utilizadas principalmente para o tratamento de morbidades menores (Dergal et al., 2002; Veiga, 2008; Feijó et al., 2012; Lima et al., 2012; Oliveira \& Menini Neto, 2012).

Com os avanços científicos, o consumo de plantas medicinais perdeu espaço para os medicamentos sintéticos. Entretanto, o alto custo destes fármacos, o difícil acesso, os seus efeitos colaterais, bem como, nos dias atuais, o uso crescente de produtos de origem natural, contribuíram para o ressurgimento do uso de plantas medicinais (Gama \& Silva, 2006; Brasileiro et al., 2008).

Considerando que diversos estudos comprovam que as plantas medicinais têm alto valor terapêutico e possuem propriedades reconhecidas de cura, prevenção, diagnóstico ou tratamento de sintomas de doenças (Arnous et al., 2005; Cunha et al, 2010; Guedes et al., 2012), seu uso é benéfico e recomendado. Porém, algumas pesquisas mostram que muitas dessas plantas possuem substâncias maléficas e, por essa razão, devem ser utilizadas com cautela, respeitando seus riscos toxicológicos (Rodrigues et al., 2011; Bochner et al., 2012; Costa et al., 2012). Assim, cuidados especiais devem ser considerados desde a coleta, secagem, armazenamento e preparação das plantas medicinais, a fim de que seus efeitos benéficos sejam atingidos (Balbach, 1993; Benedetti, 2008; Anvisa, 2010).

Neste contexto, este trabalho teve como objetivo a valorização do saber popular por meio da obtenção de informações sobre as plantas medicinais utilizadas pelos idosos do município de Marmeleiro-Paraná e, a partir de então, oferecer assistência baseada no conhecimento popular e científico a fim de que os mesmos possam utilizálas e aproveitá-las melhor, evitando resultados indesejáveis.

\section{MATERIAL E MÉTODO}

Foi realizada uma entrevista de grupo focal no mês de agosto de 2010, de acordo com as normas estabelecidas pelo Comitê de Ética em Pesquisa Envolvendo Seres Humanos (CEPEH) (número do processo 19053) da Universidade Paranaense - UNIPAR, com 35 idosos da Pastoral do Idoso do município de Marmeleiro, Sudoeste do estado do Paraná, que se reuniam uma vez por semana para desenvolverem diversas atividades. Os idosos, 25 mulheres e 10 homens, com idade acima de 60 anos, residiam na área urbana do município e eram pertencentes à classe média-baixa.
Foi apresentado, aos idosos, amostras de 20 espécies de plantas medicinais in vivo, coletadas na região urbana do município, escolhidas após levantamento bibliográfico e devido ao seu reconhecido uso pela população do município: alcachofra (Cynara scolymus L.), alecrim (Rosmarinus officinalis L.), arruda (Ruta gravenolens L.), babosa (Aloe vera L.), boldo-da-terra (Plectranthus barbatus Andrews), camomila (Matricaria chamomila L.), carqueja (Baccharis trimera Less), erva-cidreira (Melissa officinalis L.), funcho (Foeniculum vulgare Gaetn), gengibre (Zingiber officinale Roscoe), guaco (Mikania glomerata Spreng), hortelã (Mentha piperita L.), macela (Achyrocline satureoides Lam.), malva (Malva silvestris L.), manjerona (Origanum majorona L.), noz-moscada (Myristica fragans Houtt), orégano (Origanum vulgare L.), pata-de-vaca (Bauhinia forficata Link), quebra-pedra (Phyllanthus niruri L.) e sálvia (Salvia officinalis L.).

Durante a entrevista, cada espécie medicinal foi apresentada individualmente ao grupo de 35 idosos. Após cada questionamento verbal, os idosos respondiam oralmente às perguntas, que foram transcritas e analisadas posteriormente. Em seguida foi proferida uma palestra aos idosos enfatizando o modo de cultivo e colheita através de técnicas desconhecidas por eles, bem como a melhor forma de utilização de cada espécie medicinal, estimulando seu uso, e minimizando riscos de uso indevido.

\section{RESULTADO E DISCUSSÃO}

Dos idosos entrevistados, $94,3 \%$ utilizavam plantas medicinais para tratar suas enfermidades, sendo que somente $5,7 \%$ não faziam este uso. Inclusive, $71,4 \%$ utilizavam plantas medicinais frequentemente/diariamente, e somente $22,9 \%$ raramente, enquanto $5,7 \%$ nunca as empregavam. Esses resultados são corroborados com os dados encontrados no trabalho de Vigano et al. (2007), pois $82 \%$ das pessoas entrevistadas faziam uso de plantas medicinais, e mais de $50 \%$ destas as consumiam com frequência, quase que cotidianamente. $\mathrm{O}$ consumo frequente de plantas medicinais também foi mostrado em diversos trabalhos, dentre eles, o de Silva et al. (2009), Marinho et al. (2011) e Oliveira \& Menini Neto (2012).

Das plantas medicinais apresentadas aos idosos, todos (100\%) conheciam a babosa, a camomila, a erva-cidreira, a macela, a malva, a manjerona, a noz-moscada, a pata-de-vaca e a sálvia. Cerca de 94,3\% reconheceram a carqueja, $91,4 \%$ o alecrim e a hortelã, $88,6 \%$ o funcho, $85,7 \%$ a arruda e o guaco, $80 \%$ o orégano, $74,3 \%$ a alcachofra, $71,4 \%$ o boldo, $62,9 \%$ o gengibre e $51,4 \%$ a quebra-pedra, conforme Figura 1. 
De acordo com Gama \& Silva (2006), que realizaram entrevista com idosos do centro de saúde da área central da cidade de São Paulo, foram citadas mais de 30 plantas populares, sendo que as mais reconhecidas foram a camomila $(16 \%)$, a erva-cidreira e a erva-doce (14\%), apoiando os dados do presente estudo. No trabalho de Rezende \& Cocco (2002), algumas das plantas citadas como conhecidas dos residentes na área rural de Santa Rita de Calda - Minas Gerais, foram semelhantes às do presente trabalho, como macela $(24,2 \%)$, alecrim $(21,2 \%)$, erva-cidreira $(21,2 \%)$, arruda $(18,2 \%)$, hortelã $(18,2 \%)$, malva $(18,2 \%)$, quebra-pedra $(18,2 \%)$, unha-de-vaca $(18,2 \%)$ e boldo $(12,1 \%)$. Apesar disto, pode-se notar que o conhecimento de determinadas plantas medicinais difere de acordo com a região do Brasil.

Com relação à forma de preparo das plantas medicinais, a maioria dos idosos entrevistados preparava o alecrim, a babosa, a camomila, a ervacidreira, o gengibre, o guaco, a hortelã, a macela, a malva, a manjerona, a pata-de-vaca e a sálvia na forma de decocção. A carqueja, o funcho e a quebrapedra eram preparados por infusão, e a alcachofra, a arruda e o boldo na forma de maceração, conforme mostra a Figura 2. Estes dados demonstram que a maior parte das plantas medicinais era preparada de forma correta do ponto de vista técnico-científico e farmacológico (Anvisa, 2010).

Vale destacar ainda que estes dados são similares aos encontrados por Arnous et al. (2005), Pinto et al. (2006), Brasileiro et al. (2008), Silva et al. (2008), Rutkanskis \& Silva (2009), Oliveira et al. (2011) e Oliveira \& Menini Neto (2012), uma vez que os entrevistados destes trabalhos citaram o chá como a principal forma de preparo das plantas medicinais consumidas. Entretanto, em alguns casos, preparar chás (realizado pelo cozimento das espécies medicinais), de plantas que deveriam ser infundidas (em que a água fervente é vertida sobre o vegetal cortado), pode prejudicar alguma parte da planta pelo cozimento, como as folhas, flores e raízes (Rezende \& Cocco, 2002). Brasileiro et al. (2008) afirmam que o preparo das plantas medicinais para a utilização do tratamento de doenças é um ponto de grande importância para que não ocorram reações adversas. Assim, percebe-se que ações voltadas a orientação sobre o uso adequado, incluindo preparo, conservação e dosagem, devem ser fornecidas aos idosos, evitando-se assim, efeitos adversos.

Apesar disto, no presente estudo, os idosos negaram obter algum problema após o uso das plantas medicinais. De acordo com Rutkanskis \& Silva (2009), apenas $1,5 \%$ dos entrevistados apresentaram reações adversas por fazer uso de plantas medicinais, tais como enjoo e dor de cabeça, não sendo especificada a planta que causou tais reações.

As plantas medicinais mais utilizadas pelos idosos entrevistados no presente trabalho foram: o guaco $(82,9 \%)$, a erva-cidreira $(60 \%)$, a macela $(51,4 \%)$, a alcachofra $(48,6 \%)$, a pata-de-vaca $(45,7 \%)$ e a camomila e a malva $(42,9 \%)$, conforme Figura 3. Resultados de diversos trabalhos (Brandão et al., 2001; Rezende \& Cocco, 2002; Marchese et al. 2004; Arnous et al., 2005; Gama \& Silva, 2006; Veiga, 2008; Oliveira et al., 2011; Oliveira \& Menini Neto, 2012) mostram que essas plantas costumam ser as mais utilizadas pela população, pois são de uso consagrado pela medicinal tradicional.

Por outro lado, quando os idosos foram questionados sobre o uso diário das plantas medicinais, as plantas citadas foram: a erva-cidreira (48,6\%), a malva (34,3\%), a quebra-pedra (34,1\%), a sálvia $(25,7 \%)$, a camomila $(22,9 \%)$, o guaco $(20 \%)$, a carqueja e a macela (14,3\%), a pata-de-vaca e o alecrim $(8,6 \%)$ e a babosa $(2,9 \%)$. Corroborando com esses dados está o trabalho de Gama \& Silva (2006), que mostrou que são ingeridas diariamente a camomila, a erva-cidreira e a erva-doce, segundo

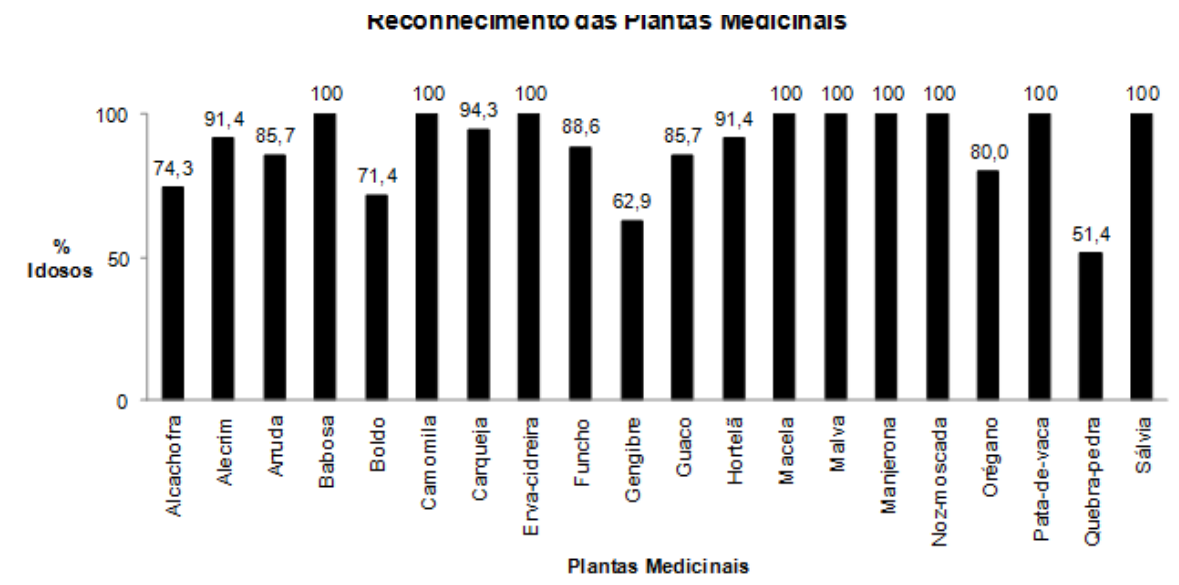

FIGURA 1. Percentual de reconhecimento das diferentes plantas medicinais apresentadas aos idosos.

Rev. Bras. PI. Med., Campinas, v.15, n.4, supl.I, p.632-638, 2013. 


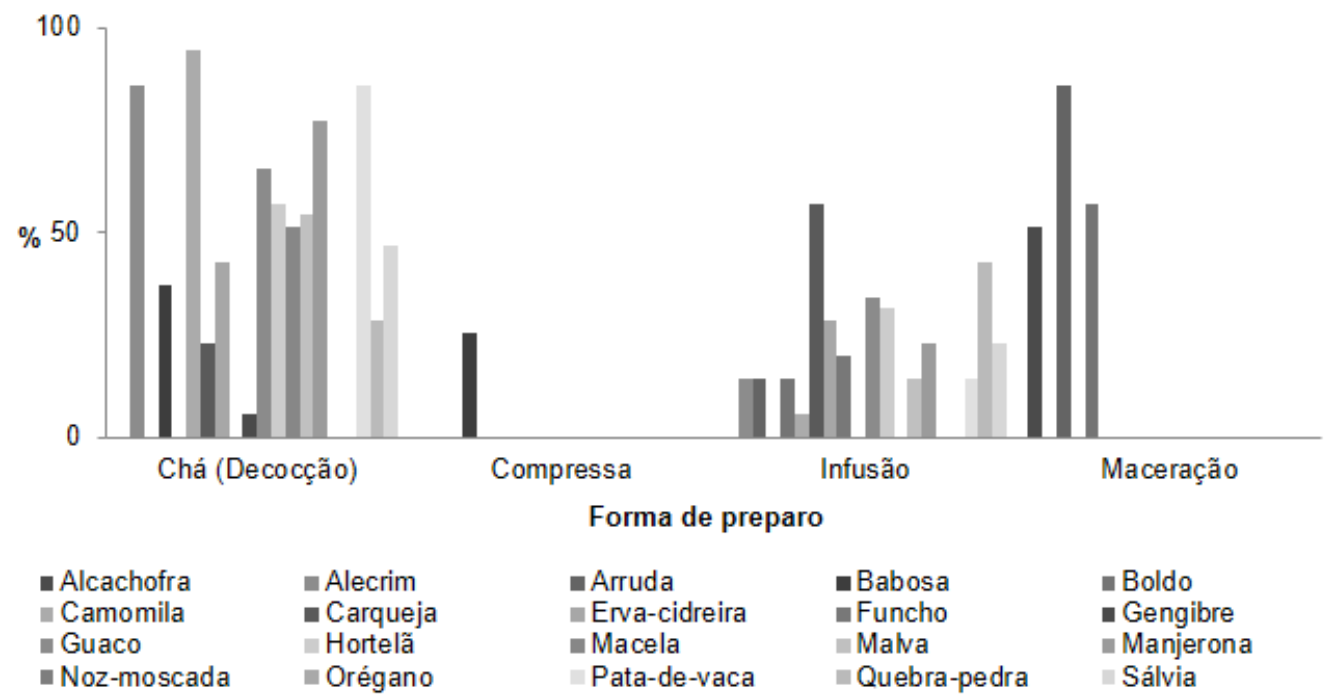

FIGURA 2. Forma de preparo das diferentes plantas medicinais de acordo com os 35 idosos pesquisados.

Uso das Plantas Medicinais

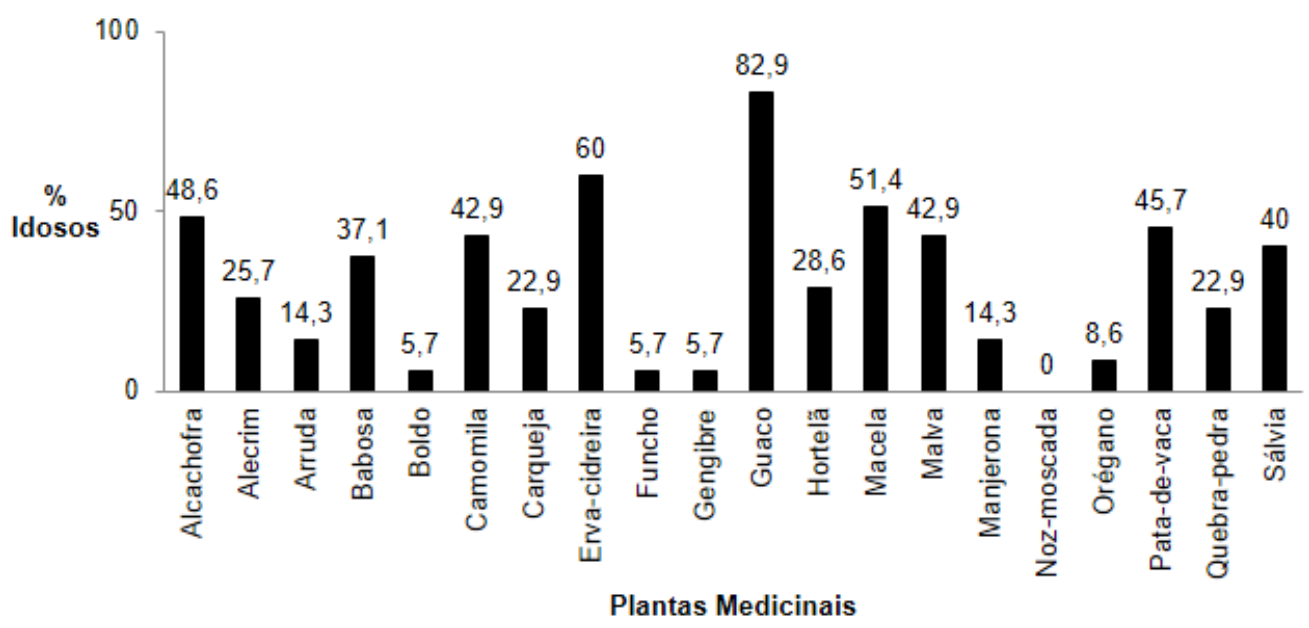

FIGURA 3. Percentual de uso das diferentes plantas medicinais pelos idosos.

eles, ingeridas como calmantes naturais.

Os fitoterápicos são medicamentos preparados exclusivamente com plantas ou partes das plantas medicinais (raízes, cascas, folhas, flores, frutos ou sementes), que possuem propriedades reconhecidas de cura, prevenção, diagnóstico ou tratamento sintomático de doenças, validadas em estudos etnofarmacológicos (Rezende \& Cocco, 2002). Isso pode ser observado no presente estudo, pois, quando o grupo de idosos foi questionado sobre o uso das plantas medicinais, e se o efeito esperado havia sido alcançado, 94,3\% afirmaram que obtiveram melhora após uso e, somente 5,7\%, disseram que não tiveram nenhum resultado.

O consumo e uso de plantas medicinais simboliza um dos aspectos importantes da cultura de um povo, sendo utilizada e difundida pelas populações ao longo de várias gerações, tanto que, quando os idosos foram questionados sobre a influência do uso de plantas medicinais, 94,2\% disseram ter aprendido seu uso com familiares (pais, avós) e os outros por indicação médica ou por influência de amigos ou, até mesmo, por lerem em livros, jornais, revistas ou na mídia, conforme Figura 4. Estes resultados corroboram com os encontrados por Gama \& Silva (2006), Veiga (2008), Macedo et al. (2007), Lima et al. (2011), Marinho et al. (2011), Oliveira \& Menini Neto (2012) e Oliveira et al. (2012).

Segundo dados levantados por Brasileiro et al. (2008), a maior parte das informações sobre a utilização e conhecimento de plantas medicinais é proveniente da tradição familiar. Segundo ele, a 
fitoterapia no Brasil sobreviveu devido às raízes profundas na consciência popular, que reconhece a eficácia e legitimidade. Os conhecimentos provenientes de gerações anteriores devem ser conservados, entretanto, é importante ressaltar que as pessoas que têm este conhecimento são aquelas com idade superior a 60 anos e nível de escolaridade mais baixo, ao passo que as pessoas mais jovens e com melhor nível de escolaridade se mostram pouco interessadas na fitoterapia.

A principal forma de obtenção das plantas medicinais relatada pelos idosos foi por plantação própria $(91,4 \%)$, seguida de mercados e farmácias, conforme Figura 5. Estes dados mostram que a utilização das plantas medicinais é facilitada pela obtenção das plantas, pois as mesmas são cultivadas pelos próprios usuários e, provavelmente, utilizadas ainda frescas. A facilidade de obtenção e utilização das plantas pelos idosos entrevistados fica evidente já que as mesmas geralmente estão disponíveis para consumo imediato.

Brasileiro et al. (2008) também confirmaram em seu estudo que, entre a população que utiliza plantas medicinais, freqüentemente $(55,47 \%)$, a obtenção das plantas é por plantações próprias, por ser mais fácil de obtê-las ainda frescas. Resultados semelhantes também foram encontrados por Oliveira \& Araujo (2007), Silva et al. (2008), Oliveira et al. (2011) e Aguiar \& Barros (2012).

\section{CONCLUSÃO}

Desta forma, os resultados do presente estudo demonstram que os idosos do município de Marmeleiro - Paraná possuem ótimo conhecimento sobre as plantas medicinais, em termos de reconhecimento e forma de preparação. Além disso, o consumo frequente das plantas medicinais, influenciado principalmente pelos familiares e pela obtenção destas em suas próprias plantações, destaca que esta cultura popular ainda prevalece nesta faixa etária e região do Brasil. Assim, informações acerca das plantas medicinais devem ser disponibilizadas para a população, em especial a mais idosa, enfatizando os modos de cultivo e colheita, através de técnicas desconhecidas por

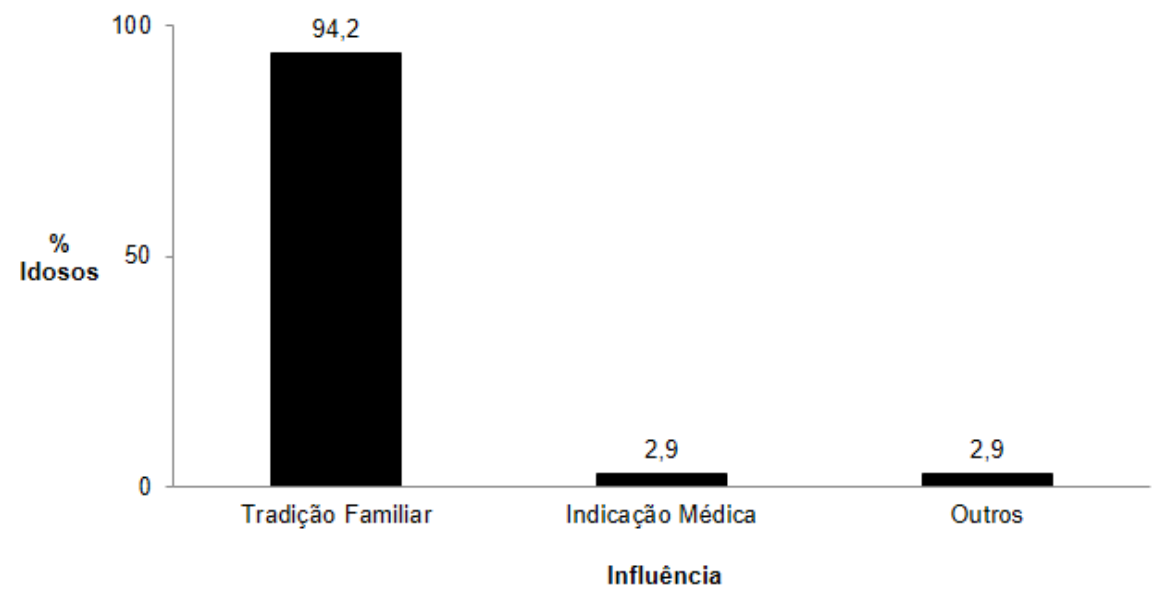

FIGURA 4. Influência da utilização das diferentes plantas medicinais pelos idosos.

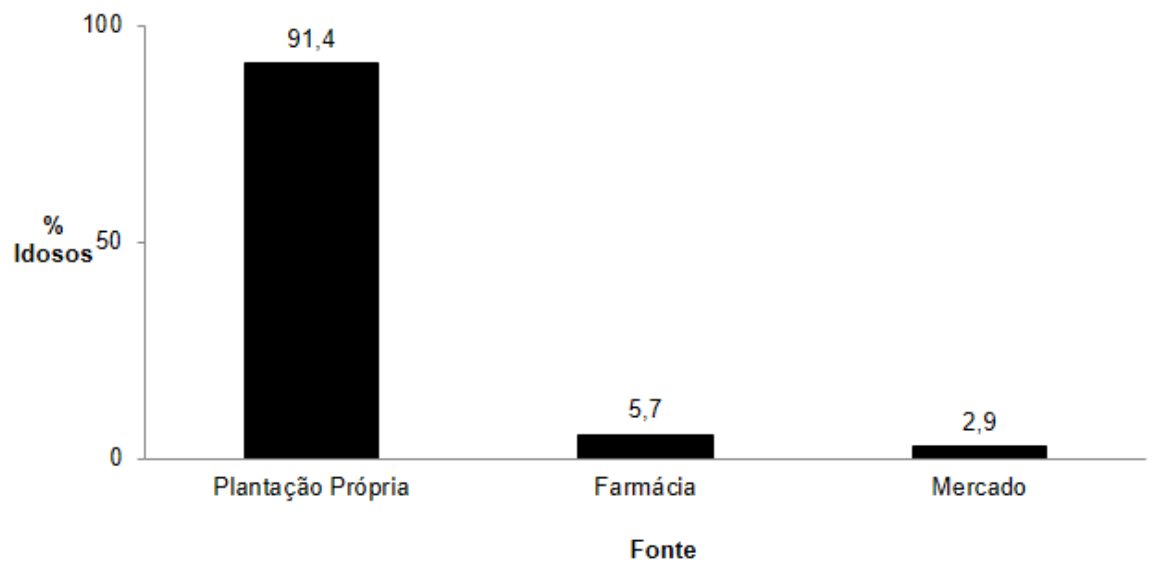

FIGURA 5. Forma de obtenção das diferentes plantas medicinais pelos idosos. 
eles, e a melhor forma de utilização de cada espécie medicinal, estimulando ainda mais seu uso, dentro de critérios que evitem prejuízos para a saúde.

\section{REFERÊNCIA}

AGÊNCIA NACIONAL DE VIGILÂNCIA SANITÁRIA (ANVISA). Resolução № 10, de 9 de março de 2010. Dispõe sobre a notificação de drogas vegetais junto à Agência Nacional de Vigilância Sanitária (ANVISA) e dá outras providências. Disponível em: <http:://www. anvisa.gov.br>. Acesso em: 21 maio 2013.

AGUIAR, L.C.G.G.; BARROS, R.F.M. Plantas medicinais cultivadas em quintais de comunidades rurais no domínio do cerrado piauiense (Município de Demerval Lobão, Piauí, Brasil). Revista Brasileira de Plantas Medicinais, v.14, n.3, p.419-434, 2012.

ARNOUS, A.H. et al. Plantas medicinais de uso caseiro, conhecimento popular e interesse por cultivo comunitário. Revista Espaço para Saúde, v.6, n.2, p.6, 2005.

BALBACH, A. As Plantas Curam. 2 ed. Itaquaquecetuba: Vida Plena, 1993.

BENEDETTI, C.B. Estudo etnobotânico de plantas medicinais na área urbana da cidade de Marmeleiro. 2008. 36p. Trabalho de Conclusão de Curso (Curso de Ciências Biológicas) - Universidade Paranaense, Francisco Beltrão.

BOCHNER, R. et al. Problemas associados ao uso de plantas medicinais comercializadas no Mercadão de Madureira, município do Rio de Janeiro, Brasil. Revista Brasileira de Plantas Medicinais, v.14, n.3, p.537547, 2012.

BRANDÃO, M.G.L. et al. Interesse dos estudantes de Farmácia e Biologia por plantas medicinais e fitoterapia. Revista Brasileira de Farmacognosia, v.11, n.2, p.7176, 2001.

BRASILEIRO, B.G. et al. Plantas medicinais utilizadas pela população atendida no programa de saúde da família de Governador Valadares-MG Brasil. Revista Brasileira de Ciências Farmacêuticas, v.44, n.4, p.629-636, 2008.

COSTA, K.C.S. Medicinal plants with teratogenic potential: current considerations. Brazilian Journal of Pharmaceutical Sciences, v.48, n.3, p.427-433, 2012.

CUNHA, A.M. et al. Hypoglycemic activity of dried extracts of Bauhinia forficata Link. Journal Phytomedicine, v.17, n.1, p.37-41, 2010.

DERGAL, J.M. et al. Potential interactions between herbal medicines and conventional drug therapies used by older adults attending a memory clinic. Drugs \& Aging, v.19, n.11, p.879-886, 2002.

FEIJÓ, A.M. et al. Plantas medicinais utilizadas por idosos com diagnóstico de Diabetes mellitus no tratamento dos sintomas da doença. Revista Brasileira de Plantas Medicinais, v.14, n.1, p.50-56, 2012.

GAMA, X.; SILVA, M.A.P. A utilização da fitoterapia por idosos de um centro de saúde em área central da cidade de São Paulo. Saúde Coletiva, v.11, n.3, p.79-84, 2006.

GUEDES, A.P. et al. Hypericum sp.: essential oil composition and biologic activities. Phytochemestry
Reviews, v.11, p.127-152, 2012.

LIMA, R.A. et al. Levantamento etnobotânico de plantas medicinais utilizadas na cidade de Vilhena, Rondônia. Revista Pesquisa \& Criação, v.10, n.2, p.165-179, 2011.

LIMA, S.C.S. et al. Representations and uses of medicinal plants in elderly men. Revista Latino-Americana de Enfermagem, v.20, n.4, p.778-786, 2012.

LOYA, A.M. et al. Prevalence of polypharmacy, polyherbacy, nutritional supplement use and potential product interactions among older adults living on the United States-Mexico border: a descriptive questionnaire-base study. Drugs \& Aging, v.26, n.5, p.423-436, 2009.

MACEDO, A.F. et al. Ocorrência do uso de plantas medicinais por moradores de um bairro do município de Marília- SP. Revista de Ciências Farmacêuticas Básica e Aplicada, v.28, n.1, p.38-45, 2007.

MARCHESE, J.A. et al. Perfil dos consumidores de plantas medicinais e condimentares do município de Pato Branco-PR. Horticultura Brasileira, v.22, n.2, p.332-335, 2004.

MARINHO, M.G.V. et al. Levantamento etnobotânico de plantas medicinais em área de caatinga no município de São José de Espinharas, Paraíba, Brasil. Revista Brasileira de Plantas Medicinais, v.13, n.2, p.170182, 2011.

OLIVEIRA, C.J.; ARAUJO, T.L. Plantas medicinais: usos e crenças de idosos portadores de hipertensão arterial. Revista Eletrônica de Enfermagem, v.09, n.1, p.93105, 2007.

OLIVEIRA, E.R.; MENINI NETO, L. Levantamento etnobotânico de plantas medicinais utilizadas pelos moradores do povoado de Manejo, Lima Duarte - MG. Revista Brasileira de Plantas Medicinais, v.14, n.2, p.311-320, 2012.

OLIVEIRA, L.S. et al. Plantas Medicinais como Recurso Terapêutico em Comunidade do Entorno da Reserva Biológica do Tinguá, RJ, Brasil - Metabólitos Secundários e Aspectos Farmacológicos. Revista Científica Internacional, v.4, n.17, p.54-74, 2011.

OLIVEIRA, S.G.D. et al. An ethnomedicinal survey on phytotherapy with professionals and patients from Basic Care Units in the Brazilian Unified Health System. Journal of Ethnopharmacology, v.140, n.2, p.428437, 2012

PINTO, E.P.P. et al. Conhecimento popular sobre plantas medicinais em comunidades rurais de mata atlântica Itacaré -BA, Brasil. Acta Botanica Brasilica, v.20, n.4, p.751-762, 2006.

REZENDE, H.A.; COCCO, M.I.M. A utilização de fitoterapia no cotidiano de uma população rural. Revista da Escola de Enfermagem - USP, v.36, n.3, p.282-288, 2002.

RITTER, M.R. et al. Plantas usadas como medicinais no município de Ipê, RS, Brasil. Revista Brasileira de Farmacognosia, v.12, n.2, p.51-62, 2002.

RODRIGUES, H.G. et al. Efeito embriotóxico, teratogênico e abortivo de plantas medicinais. Revista Brasileira de Plantas Medicinais, v.13, n.3, p.359-366, 2011.

RUTKANSKIS, A.M.R.A.; SILVA, C.T.A.C. Utilização de plantas medicinais pelos acadêmicos da área de saúde da Faculdade Assis Gurgacz no município de Cascavel Paraná. Cultivando o Saber Cascavel, v.2, n.4, p.69-85, 2009.

Rev. Bras. PI. Med., Campinas, v.15, n.4, supl.I, p.632-638, 2013. 
SILVA, F.L.A. et al. Use of medicinal plants by the elders at a family's health estrategy. Revista de Enfermagem, v.2, n.1, p.9-16, 2008.

SILVA, M.D. et al. Estudo etnobotânico de plantas medicinais utilizadas pela população rural no entorno do Parque Nacional da Serra do Itajaí - Indaial. Revista Saúde e Ambiente, v.10, n.2, p.54-64, 2009.

VIGANO, J. et al. Utilização de plantas medicinais pela população da região urbana de Três Barras do Paraná. Acta Scientarium Health Sciences, v.29, n.1, p.27-36, 2007.

VEIGA, V.F. Estudo do consumo de plantas medicinais na região Centro Norte do Estado do Rio de Janeiro: aceitação pelos profissionais de saúde e modo de uso pela população. Revista Brasileira de Farmacognosia, v.18, n.2, p.308-13, 2008. 